\title{
Social and Behavioural Determinants of the Difference in Survival among Older Adults in Japan and England
}

\author{
Jun Aida ${ }^{a, b}$ Noriko Cable ${ }^{c}$ Paola Zaninotto ${ }^{c}$ Toru Tsuboya $^{b}$ \\ Georgios Tsakos ${ }^{c}$ Yusuke Matsuyama $^{b}$ Kanade Ito $^{d}$ Ken Osaka $^{b}$ \\ Katsunori Kondo ${ }^{e}, f$ Michael G. Marmot ${ }^{c}$ Richard G. Watt ${ }^{c}$ \\ ${ }^{a}$ Center for Epidemiology, Biostatistics and Clinical Research, Tohoku University Graduate School of Dentistry, \\ Aoba-ku, Sendai, Japan; ${ }^{b}$ Department of International and Community Oral Health, Tohoku University Graduate

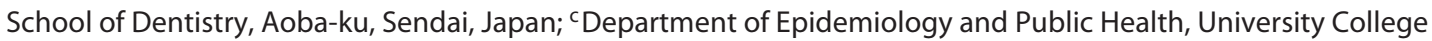 \\ London, London, UK; ${ }^{d}$ Division of Oral Health Sciences, Department of Health Sciences, School of Health and \\ Social Services, Saitama Prefectural University, Koshigaya, Japan; ${ }^{e}$ Center for Preventive Medical Sciences, \\ Chiba University, Chiba, Japan; ${ }^{\mathrm{C} C e n t e r}$ for Gerontology and Social Science, National Center for Geriatrics and \\ Gerontology, Obu, Japan
}

\section{Keywords}

Cross-country comparative study - Laplace regression .

Cohort study $\cdot$ Mortality $\cdot$ Social relationships

\begin{abstract}
Background: A rapidly ageing population presents major challenges to health and social care services. Cross-country comparative studies on survival among older adults are limited. In addition, Japan, the country with the longest life expectancy, is rarely included in these cross-country comparisons. Objective: We examined the relative contributions of social and behavioural factors on the differences in survival among older people in Japan and England. Methods: We used data from the Japan Gerontological Evaluation Study (JAGES; $n=13,176$ ) and the English Longitudinal Study of Ageing (ELSA; $n=5,551)$ to analyse all-cause mortality up to 9.4 years from the baseline. Applying Laplace regression models, the 15th survival percentile difference was estimated. Results: During the follow-up, $31.3 \%$ of women and
\end{abstract}

\section{KARGER}

E-Mail karger@karger.com www.karger.com/ger
This article is licensed under the Creative Commons Attribution NonCommercial-NoDerivatives 4.0 International License (CC BYNC-ND) (http://www.karger.com/Services/OpenAccessLicense) Usage and distribution for commercial purposes as well as any distribution of modified material requires written permission.
$38.6 \%$ of men in the ELSA died, whereas $19.3 \%$ of women and $31.3 \%$ of men in the JAGES died. After adjusting for age and baseline health status, JAGES participants had longer survival than ELSA participants by 318.8 days for women and by 131.6 days for men. Family-based social relationships contributed to 105.4 days longer survival in JAGES than ELSA men. Fewer friendship-based social relationships shortened the JAGES men's survival by 45.4 days compared to ELSA men. Currently not being a smoker contributed to longer survival for JAGES women (197.7 days) and ELSA men (46.6 days), and having lower BMI reduced the survival of JAGES participants by 129.0 days for women and by 212.2 days for men. Conclusion: Compared to participants in England, Japanese older people lived longer mainly because of nonsmoking for women and family-based social relationships for men. In contrast, a lower rate of underweight, men's better friendship-based social relationships, and a lower smoking rate contributed to survival among participants in England.

(C) 2018 The Author(s)

Published by S. Karger AG, Basel 


\section{Introduction}

Overall, Japanese people have the longest life expectancy in the world, especially Japanese women [1]. Research exploring the reasons for this greater longevity among the Japanese population has identified a range of potential explanations [2-5]. A milestone review by Marmot and Smith [5] identified economic growth, a low-fat diet, the public health system, and job security in Japan as the most important factors for greater longevity in this population. More recently, "a culture of hygiene, high levels of educational attainment, an egalitarian society, and strong government that led public health programmes, particularly for tuberculosis control" [3] have been proposed as key factors explaining the longevity of the Japanese population. On the other hand, high blood pressure, higher smoking rates in men, increasing social inequalities, and increasing obesity have been reported as recent threats to Japanese longevity $[2,3]$.

The health of the older population has become more important in ageing societies, and life expectancy at birth does not necessarily correspond to life expectancy at older age [6]. To date, there is limited evidence from crosscountry comparative studies that have directly examined the respective roles of health, behavioural, and social factors in relation to longevity among older adults. One study examined the association between behavioural factors and life expectancy among older adults though it focused on European countries only [7]. However, most of the cross-country comparison studies on mortality only included Western countries. Here we compared population data from Japan, the country with the highest life expectancy, with population data from England. The aim of this prospective cohort study was to determine the relative contributions of social and behavioural factors to differences in survival between older adults in England and Japan.

\section{Methods}

\section{Data Source}

Cohort data from two on-going prospective cohort studies, the English Longitudinal Study of Ageing (ELSA) [8,9] and the Japan Gerontological Evaluation Study (JAGES) Project [10, 11], were used in this analysis. ELSA targets independent-living older adults in England aged 50 years or older and the first wave was conducted between 2002 and 2003. The first survey of the JAGES Project was conducted in 2003, drawing participants from 6 municipalities in the Aichi prefecture (at the time, the project was named the Aichi Gerontological Evaluation Study [AGES]). Although Japan has Okinawa, known in the past years as the Blue Zone [12], which is characterised as longer life in the world, the baseline survey did not cover this area. JAGES participants were community-dwelling individuals aged 65 years or older that were randomly selected from each municipality. Further details of ELSA and JAGES can be found in each cohort profile $[8,10]$. In our study, we analysed the data from individuals aged 65 years or older at the first wave in both studies.

\section{Ethical Considerations}

Ethical approval for the ELSA was granted from the Multicentre Research and Ethics Committee (MREC/01/2/91). The AGES and JAGES study protocol and informed consent procedure were approved by the Ethics Committee on the Research of Human Subjects at Nihon Fukushi University (04-05 and 10-05).

\section{Outcome Measure}

The outcome was all-cause mortality occurring until 9.4 years (3,436 days) from baseline. The end of follow-up was March 15, 2012 for the ELSA and March 28, 2013 for the JAGES. For both studies, mortality data were obtained by linking with administrative records. For the ELSA, all-cause mortality was ascertained for the consenting study members ( $95 \%$ of the eligible participants) by linking to the National Health Service mortality register. For the JAGES, the mortality records of the national long-term care insurance database (which contains each death reported by physicians to their local municipal government) were linked with $99 \%$ of the cohort participants.

\section{Behavioural and Social Factors}

For health-related behaviours, we used physical activity, smoking, alcohol drinking, and body mass index (BMI). A level of physical activity was indicated by self-report on participants' active involvement in a sports club. The response was dichotomised. Based on the self-reported smoking status, participants were categorised into never smokers, past smokers, or current smokers. For alcohol drinking, participants were categorised into: non-drinking, not drinking every day, or drinking every day. A previous study reported that a BMI of 22.5-25.0 had the lowest risk of mortality [13]; we set a BMI of 22.5-24.9 as the reference category of BMI variable. In our data, a small number of Japanese participants had a BMI of 30 or over, participants with a BMI of 25 or over were put into a category. Then, BMI was divided into the following groups: less than 18.5, between 18.5 and 22.4, between 22.5 and 24.9 , or 25 or over.

For social factors, socioeconomic status and social relationships were used. As an indicator for socioeconomic status, participants' age when they completed their formal education (15 years or younger, 16-18 years, 19 years or older) was used for both countries. In our study, social relationships were assessed via family (marital status) and friendship-based social relationships (social network). For family-based social relationships, marital status was categorised into married, divorced/widowed, or non-married. For friendship-based social relationships, we assessed the frequency of meeting up with friends, grouped into: once or more a week, once or twice a month, or less than once a month.

\section{Covariates}

Age at baseline was categorised as 65-69, 70-74, 75-79, or 85 years or older. In JAGES, older adults receiving the public disability insurance benefit were not invited to the study even if they were
Differences in Survival among Older

Adults in Japan and England
Gerontology 2018;64:266-277

DOI: $10.1159 / 000485797$
267 


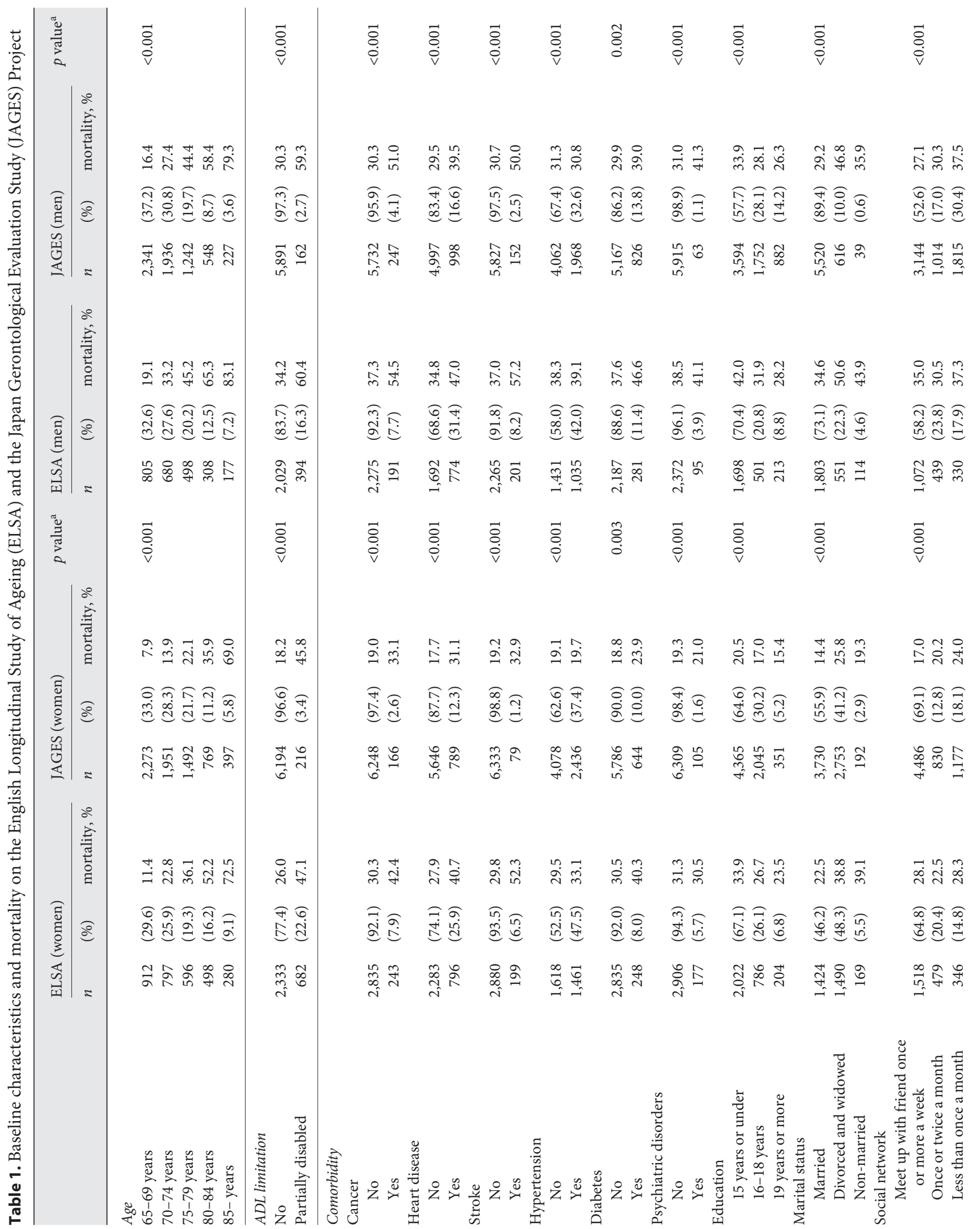




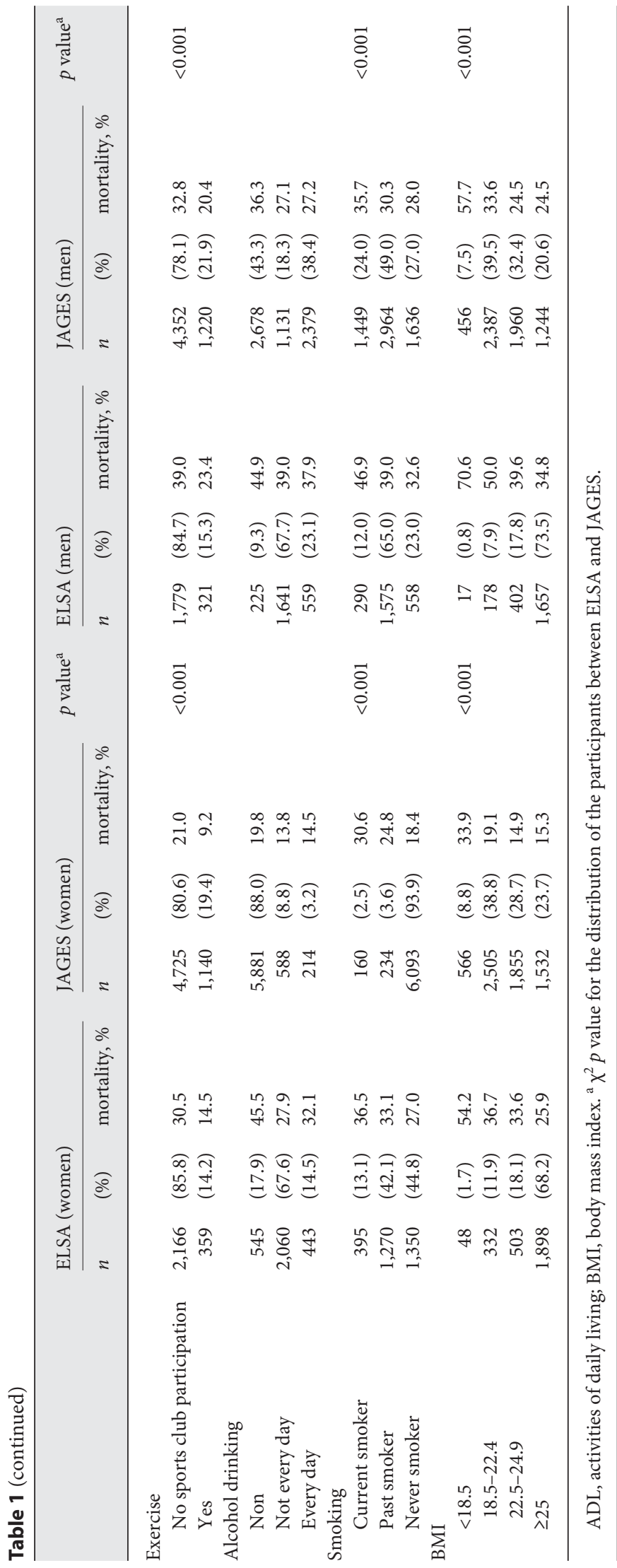

Differences in Survival among Older

Adults in Japan and England living at their home [10], whereas ELSA targets independently living older adults. Due to this research design, it is likely that systematic differences exist in participants' disability status between two studies. To account for this study design factor, activities of daily living (ADL) were adjusted in all models as covariates. ADL were determined from the participants' response to three questions addressing the presence of difficulties in walking, bathing or showering, or using the toilet in both studies. Participants who reported one or more difficulties were regarded as partially disabled. We also used comorbidities to indicate the baseline health status of the participants as covariates. The ELSA data contained self-reports of doctor-diagnosed cancer, heart disease, stroke, hypertension, diabetes, and psychiatric disorders, whereas we used the receipt of medical treatments for cancer, heart disease, stroke, hypertension, diabetes, and psychiatric disorders for the JAGES sample.

\section{Analysis}

To estimate the country differences in survival, the Laplace regression analysis was applied. The Laplace regression analysis enables the estimation of the percentiles of survival time [14-17]. In our study, the lowest mortality was observed among the female participants of JAGES at $18.5 \%$. Therefore, we used the 15th survival percentile difference (15th PD), i.e., the difference in the time when the first $15 \%$ of the participants had died, to estimate the difference in survival between the groups [17]. We also estimated the country difference in survival in relation to the explanatory factors. For sensitivity analysis, we also ran the Cox proportional hazards regression analyses $[16,17]$.

At first, to examine the contributions of each factor separately among the ELSA and JAGES participants, stratified analyses in each cohort were conducted. Then, the datasets for each cohort were combined, and we conducted pooled analyses. In the pooled analyses, we included the country dummy variable as a fixed effect to calculate the difference in survival between the ELSA and JAGES participants, and then examined the factors contributing to the difference in survival between the two cohorts, by calculating the number of days attributable to the different factors. For this purpose, several models were constructed. At first, before examining the contribution of each factor, age, ADL, and baseline health status (i.e., comorbidities) were included to adjust (Model 1). In Models 2-4, socioeconomic status (i.e., age at which the participants left full-time education) and social relationships variables (family-based and friendship-based) were separately added to Model 1 . Then, these 3 variables were simultaneously included (Model 5). Modifiable health risks were separately added for: exercise (Model 6), alcohol drinking status (Model 7), smoking status (Model 8), and BMI (Model 9). To evaluate the degree of explained difference between the countries in survival time (days) by each factor, the change in the differences in survival between the models was calculated and displayed in a graph.

All analyses were stratified by gender. We did not apply weights on our analyses, given that ELSA and JAGES used the different sample weighting strategy. To support our decision, we conducted sensitivity analysis applying weights on both datasets. Because the results showed very close findings (see online suppl. Tables 1 and 2; see www.karger.com/doi/10.1159/000485797 for all online suppl. material), we report non-weighted results. Although the mortality outcome did not have any missing information, missing data because of item non-response were dealt with multiple imputation using chained equations by the STATA programme [18]. The 
numbers of missing responses are shown in online supplementary Table 3. Imputation was conducted for each dataset (country) and gender separately and 10 multiply imputed datasets were produced. Models were independently applied for each of the $10 \mathrm{im}$ puted datasets. The imputation models included auxiliary variables known to be predictive of missingness. Then, single mean estimates and adjusted standard errors were calculated using $\mathrm{Ru}-$ bin's rule [19]. For sensitivity analysis, complete case analysis was also performed (in online suppl. Tables 4 and 5), which revealed similar results to those from the imputed data. As other sensitivity analysis, we excluded data from participants who died or dropped out during the first 2 years from the baseline to reduce the possibility of reverse causation (in online suppl. Tables 6 and 7). STATA SE version 14.1 (Stata Corp., College Station, TX, USA) was utilised for all analyses.

\section{Results}

We analysed the data obtained from 3,083 women and 2,468 men in ELSA (mean age: 74.7 [SD = 7.3] and 73.7 $[\mathrm{SD}=6.7]$ years, respectively) and 6,882 women and 6,294 men in JAGES (mean age: $73.4[\mathrm{SD}=6.3]$ and 72.5 $[\mathrm{SD}=5.7]$ years, respectively). The maximum follow-up period was 3,435 days (9.4 years), and the mean follow-up period was 3,047 days for women and 2,871 days for men, respectively.

Table 1 shows the baseline characteristics and mortality in both cohorts, and reveals significant differences between the countries in these characteristics. The number of deaths that occurred between the baseline and the end of the follow-up period was 964 (31.3\%) women and 953 $(38.6 \%)$ men in ELSA and 1,330 (19.3\%) women and 1,968 (31.3\%) men in JAGES. Mortality rates per personyear were 3.9 and $5.1 \%$ for ELSA women and men and 2.3 and 3.9\% for JAGES women and men, respectively.

The results of the univariate and multivariable Laplace regression analyses on the differences in the 15th percentile of survival according to the explanatory variables are presented separately for women (Table 2) and men (Table 3). Older age, partial disability, and comorbid status were associated with a smaller percentile of survival time in both ELSA and JAGES in the univariate and fully adjusted multivariate models. Participants with the single status had a smaller percentile of survival especially for ELSA men. Poorer friendship-based social relationships were associated with a smaller percentile of survival especially in JAGES men. More years of education, any drinking, and not being a current smoker tended to be associated with longer survival in both the ELSA and JAGES participants. Lower $(<18.5)$ BMI was associated with shorter survival especially for JAGES participants. The associa- tions between each variable and 15th PD were similar in both men and women. These associations are similar to the sensitivity analyses through Laplace regression models that excluded those who died or dropped out during 1 year or 2 years from the baseline (in online suppl. Tables 6 and 7) and Cox proportional hazards models (in online suppl. Table 8).

There were differences in survival between the JAGES and ELSA participants. After adjusting for age, ADL, and baseline health status and comparing the samples when the first $15 \%$ of the participants had died, JAGES women survived for 318.8 more days than ELSA women (in online suppl. Table 9, Model 1), and JAGES men survived for 131.6 more days than ELSA men (in online suppl. Table 10, Model 1).

Figure 1 shows how much each of the aforementioned factors explains these differences in 15 th percentile of survival between the two cohorts. For example, smoking contributed to 197.7 days (obtained from online suppl. Table 9, Models 5 and $8 ; 320.8$ minus 123.1) of longer survival for JAGES women compared to ELSA women. This is explained as follows. There were more smokers among ELSA women compared to JAGES women (Table 1), and smokers had shorter survival than non-smokers (Table 2, online suppl. Table 9). In contrast, smoking was more prevalent among JAGES men compared to ELSA men. As a result, although smoking was associated with increased mortality in both JAGES and ELSA men (Table 3), the lower smoking rate among ELSA men contributed to 46.6 days longer survival compared to JAGES men.

Among women, not smoking were the most important factor for survival among JAGES compared to ELSA participants, whereas being a non-daily drinker was the most important contributing factor for longer survival among ELSA compared to JAGES women. Not being underweight also contributed to longer survival among ELSA compared to JAGES women.

Among men, rich family-based social relationships in JAGES was the most important contributor to longer survival compared to ELSA. Although the association of family-based social relationships seemed to be stronger among ELSA men (Table 3), the prevalence of being married was higher among JAGES men (Table 1). Therefore, a higher prevalence of being married seemed to contribute to 105.4 days longer survival in JAGES compared to ELSA.

For ELSA men, not being underweight, non-daily drinking, being a non-smoker, and better friendshipbased social relationships contributed to increased survival compared to JAGES men. Friendship-based social
Aida et al. 


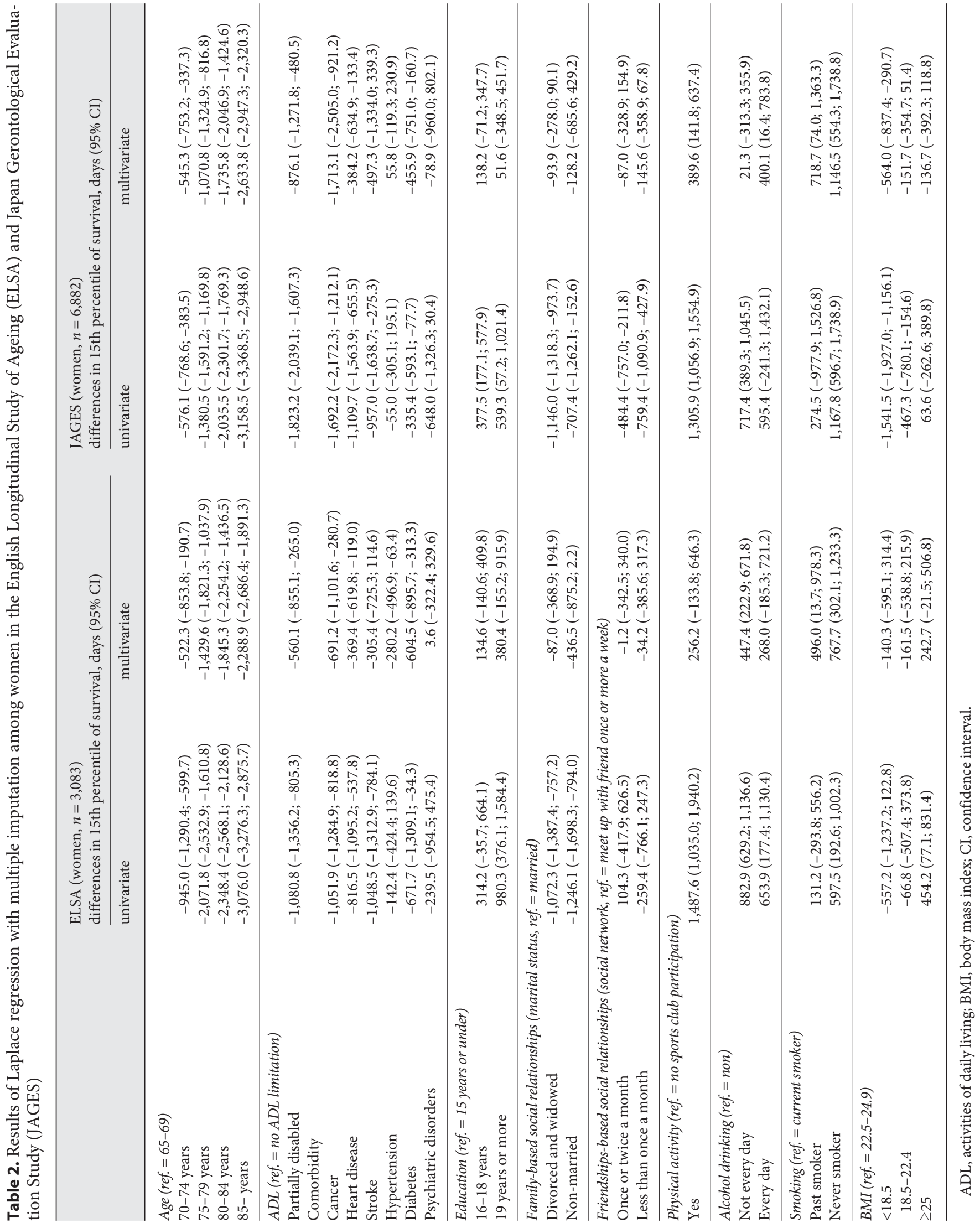




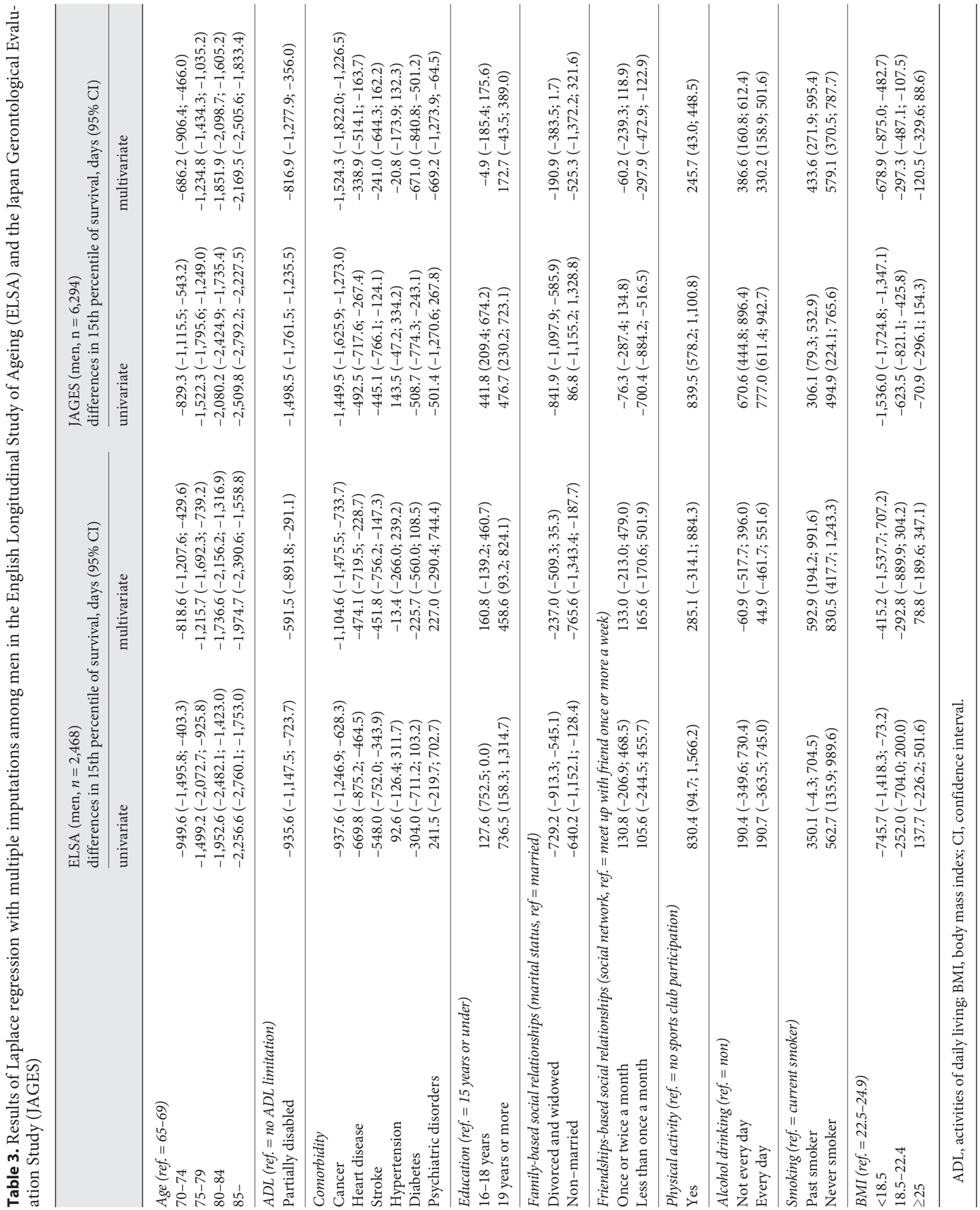




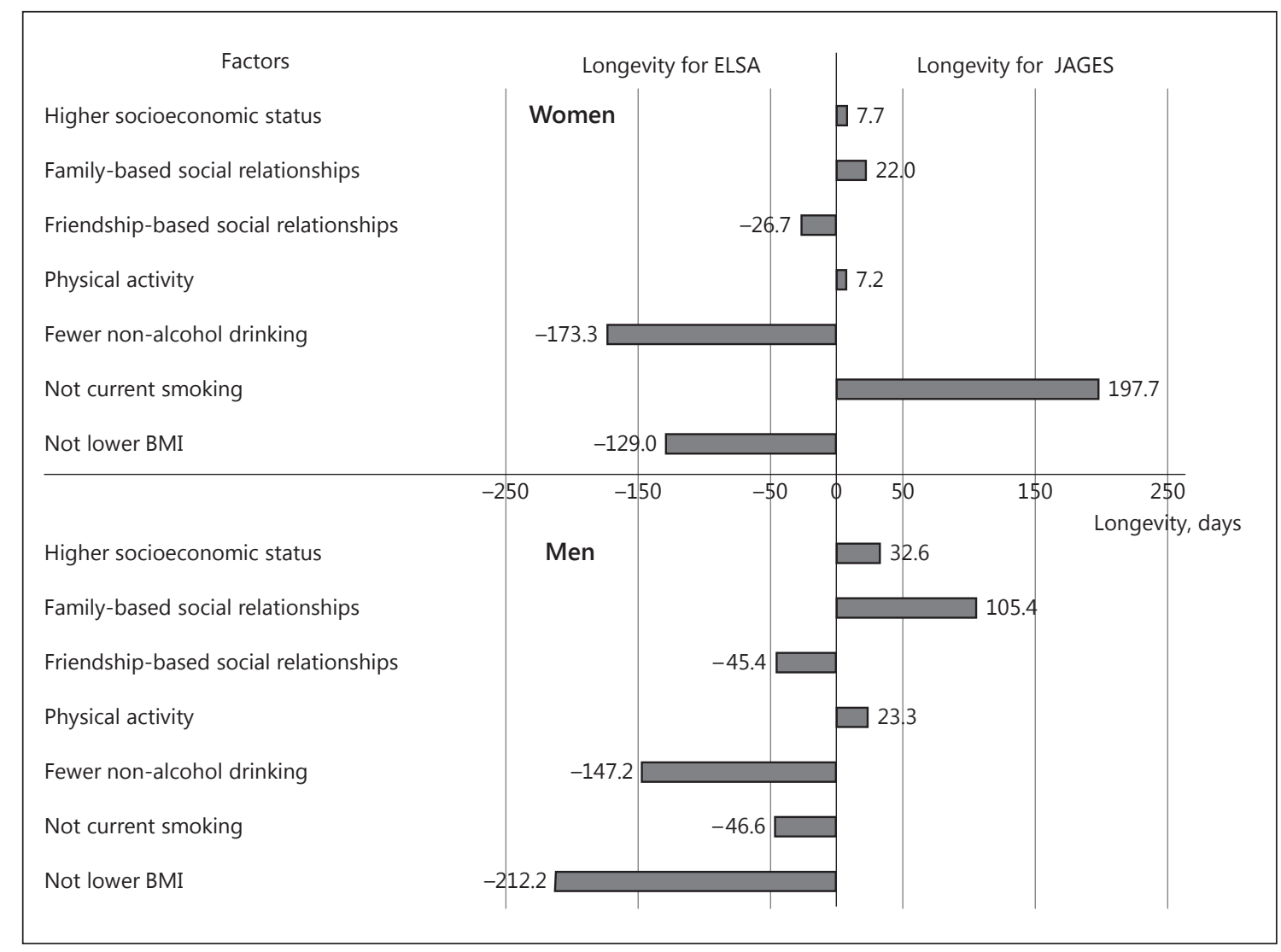

Fig. 1. Reasons for the difference in longevity (days) by each factor between the English Longitudinal Study of Ageing (ELSA) and the Japan Gerontological Evaluation Study (JAGES) presented separately by gender. Explained difference in longevity (days) was calculated from the results in online Supplementary Tables 9 and 10: socioeconomic status (model 1 minus model 2), family-based so-

relationships predicted survival among JAGES participants only (Table 3), and the prevalence of poorer friendship-based social relationships was higher among JAGES men (Table 1). Therefore, a lack of friends contributed to 45.4 fewer days of survival in JAGES compared to ELSA men.

\section{Discussion}

Research evidence on survival among older adults based on cross-country comparative studies is limited, especially with Japan, which has the longest life expectancy. In this study, we examined the differences in 15 th percentile of survival between older individuals living in Japan and England from two prospective cohort studies, and identified the factors contributing to these differences. cial relationships (model 1 minus model 3), friendships-based social relationships (model 1 minus model 4), physical activity (model 5 minus model 6), alcohol drinking (model 5 minus model 7), smoking (model 5 minus model 8), and BMI (model 5 minus model 9).

The participants in Japan lived longer than those in England at the 15th percentile of all-cause mortality after adjusting for age, ADL, and baseline health status. Major contributing factors for the higher survival among the JAGES compared to ELSA participants were lower smoking rate in women, and better family social relationships in men. In contrast, several factors contributed to longer survival in ELSA compared to JAGES participants: a lower rate of underweight, moderate alcohol drinking for both women and men, better friendship-based social relationships in men, and a lower smoking rate in men.

\section{Strengths and Limitations of this Study}

To the best of our knowledge, this is the first crosscountry comparative population-level study to show the absolute difference in survival (days) between the two countries and the size of the effect of the factors contrib- 
uting to that difference. Using absolute measure is more appropriate in public health decision making because they are easier to explain to the general public than relative risks $[20,21]$. Several previous studies directly compared country differences using pooled data, but they provided relative measures rather than absolute measures $[22,23]$. Traditional life table methods enable the direct assessment of absolute longevity, yet a limited number of factors can be tested with these methods $[6,24,25]$. Although recently applied advanced methods enable the consideration of multiple factors with absolute longevity, direct comparison between countries using pooled data has not been conducted [7].

In this study, because we could use pooled cohort data, the country in which the participant resided was treated as a fixed effect, using a dummy variable. As there is lower possibility of reverse temporal association between the country of residence among older people and individual sociodemographic characteristics and behaviours, the possibility of bias when estimating the mediation effect is small.

In both ELSA and JAGES, the mortality information was reliable and obtained via credible official sources. Additionally, most of the explanatory variables used in this analysis were obtained through a similar approach across the two studies. On the other hand, while we had relevant information for when participants were older adults, we could not consider various demographic, psychosocial, and environmental factors in early life due to a lack of available and comparable data.

There are notable differences between the two countries in those factors and they could have played a role in explaining our results; for example, accumulation of stress through divergent historical experiences could have affected the difference in health between the two cohorts. Inclusion of objective measures to indicate physical health status or environmental factors that are comparable in two cohorts could elaborate explaining the differences in the mortality between the two cohorts. Although both England and Japan have universally accessible health care systems, there are differences in health care provision [26]. The effect of medical care access on the outcome should be considered in future studies.

High blood pressure is reportedly the greatest risk factor for mortality among Japanese women [2]. Although we used comorbidities including hypertension in our models, having an objective blood pressure measure could have allowed further explanation of the differences in longevity between the two countries. In addition, because this study used cohorts of older adults who survived at least at the baseline of the survey, selection bias could overestimate the association of factors such as moderate alcohol drinking [27]. Our results suggest that moderate drinkers have a longer life than non-drinkers.

Although a J-shaped relationship between alcohol consumption and all-cause mortality has been previously reported [28], a recent systematic review has challenged the merits of moderate drinking after considering several biases [29]. In addition, a study on the British cohort of people born in 1958 suggested that non-drinking would be likely to be associated with long-term illnesses in early life [30]. Although we accounted for comorbidities at baseline, a precise measure to indicate quantity of alcohol intake that was comparable to both countries was impossible to obtain; instead, we used frequency of alcohol use. Results from alcohol use need to be carefully interpreted.

Another limitation relates to differences in the study designs between the two cohorts. The sampling strategies were different and the JAGES 2003 did not contain (or aim for) a national representative sample. In addition, older adults with disability were excluded from the JAGES but not from the ELSA sample. We accommodated the potential selection bias by accounting for the baseline ADL and we only included those aged 65 years and over in both studies. Additionally, in the sensitivity analyses, we excluded participants who died within 1 year or 2 years from the baseline, which likely reduced the possibility of health selection bias in our study.

Our study is based on the use of self-reported questionnaires from two cohorts. It is possible that comparability in these cohorts is limited to some degree, such as ADL. Given the cultural differences in these two cohorts, their response to the ADL questions may be different. However, the questions were phrased similarly in the two cohorts with regard to their ability to perform certain daily tasks. Moreover, this is treated as a covariate in our study. We are therefore confident that potential response bias is minimal in our study. Questions were phrased differently to the cohort members to obtain baseline health status for this study, which raises the question for comparability in this study. However, baseline health status is treated as a covariate in the model; we think that the effect on the longitudinal associations between social determinants and longevity is minimal.

In relation to the analyses, we estimated the 15th survival $P D$, the difference in the time when the first $15 \%$ of the participants had died. Future studies with longer follow-up would better inform cross-country differences in social determinants of longevity. 
Although the effect of the long-term change in the baseline characteristics on longevity is not in our study focus, this is likely to affect the course of longevity. Future studies including time-varying factors would inform us how the policies relevant to healthy ageing to be formulated.

\section{Comparison with Previous Studies}

The reasons for the longevity observed in Japanese people have been examined in previous studies. The life expectancy at birth for Japanese women was the longest internationally with 86.8 years in 2015 [1]. Japanese men also have a comparatively long life expectancy at 80.5 years. Since the milestone studies related to longevity among Japanese individuals conducted by Marmot and his colleagues $[4,5]$, lower mortality from coronary heart disease, which is partly attributable to lower serum cholesterol levels, has been considered one of the reasons for the longer life expectancy found among the Japanese [3]. However, present analyses did not show any significant association of overweight, a possible indication of higher serum low density cholesterol, and therefore shorter survival. On the contrary, we found a significant association between underweight and shorter survival among Japanese participants, which can offer additional support to a J-shaped or U-shaped association between BMI and mortality among Japanese $[12,30]$. Previous studies have reported that BMI has a J-shaped or U-shaped relationship with mortality $[13,31]$. A recent study that pooled cohort data from 10 million participants reported that those with a BMI of 22.5-25.0 had the lowest risk of death compared to those who were categorised as underweight or overweight [13]. Findings from our previous study on older Japanese individuals also support the same trend [32].

In addition, the association of BMI with all-cause mortality varies according to age; a relatively higher BMI is associated with lower mortality among older populations compared to younger populations $[33,34]$. In a metaanalysis of older adults, higher all-cause mortality was observed among people with a BMI of 35 or more and a significant increment of hazard ratio was not observed among people with a BMI of 30-34 [34]. This may explain why we did not observe shorter survival among the overweight participants in ELSA. Being underweight among older adults is associated with frailty [35], and frailty is linked to shorter longevity [36]. In the present study, a relatively large number of older Japanese had a BMI of less than 18.5, which was associated with increased mortality. Therefore, being underweight in older populations should be considered an important public health issue, especially in Japan.

Differences in Survival among Older

Adults in Japan and England
Our results also showed that smoking reduced longevity by about 2 years even when making comparisons only for the period that the first $15 \%$ of participants died. Smoking is the greatest risk factor for death among Japanese men $[2,37]$. We also confirmed the harmful effects of smoking, as the high smoking rates among Japanese men reduced their survival and decreased the difference in survival between the Japanese and English cohorts by 46.6 days. In contrast, the lower rates of smoking among women in Japan partially explained why their survival was 197.7 days longer than women in England. Therefore, among older populations, smoking is still an important public health issue that predominantly affects different genders in these two cohorts (Japanese men and English women).

Previous studies have shown the considerable health benefits of social relationships, such as marriage and contact with friends, with both factors associated with longer longevity $[38,39]$. Our study added the knowledge about the different distribution of social relationships regarding health in two cohorts. The longevity of the participants in Japan could partly be explained by social relationships with families, especially for men. In contrast, longevity for men in England compared to men in Japan was partly derived from their social relationships with friends.

Similar to previous studies, the present study also confirmed that a higher educational level was associated with longer survival [40, 41]. However, education did not substantially explain the differences in longevity between the two cohorts of older adults, since their education levels were similar. This similar distribution of educational attainment in this generation in Japan and UK has already mentioned in a previous study [5].

\section{Public Health Implications}

Although the mean longevity was longer among the participants in Japan, we found some factors that contributed to survival among the participants in England. Tackling the factors that contribute to reduced survival, such as smoking for JAGES men and ELSA women, could effectively improve survival in both countries. In relation to social relationships, public health interventions to promote social relationships with friends have been advocated [42]. In Taketoyo in Japan, a resident-centred community intervention programme was developed to provide opportunities to promote social interactions with community members. The programme improved social interactions and reduced the incidence of functional disability among older adults. Improving social relationships with family members, skills in handling worries and

Gerontology 2018;64:266-277 DOI: $10.1159 / 000485797$
275 
demands, and conflict management within families are all potentially important components of community interventions [43].

\section{Conclusions}

The participants in Japan lived significantly longer than those in England. Lower smoking rates in women, and better family social relationships in men were major contributors to the longevity among the Japanese participants. In contrast, a lower rate of underweight, better friendship-based social relationships in men, and a lower smoking rate in men contributed to survival among participants in England. Modifying health-related behaviours and enriching social relationships could make a difference in survival among elderly at the population level.

\section{Acknowledgments}

This study was supported by a grant of the Strategic Research Foundation Grant-aided Project for Private Universities from the Ministry of Education, Culture, Sport, Science, and Technology, Japan (MEXT, http://www.mext.go.jp/en/), 2009-2013, for the Center for Well-being and Society, Nihon Fukushi University, Grants-in-Aid for Scientific Research (22330172, 22390400,
22390400, 22592327, 23243070, 23590786, 23790710, 24390469, 24530698, 24653150, 24683018, 25253052, 25870573, 25870881, 26285138, 26882010, 15H04781, 15H01972, 16H05556, 16K19267) from the Japan Society for the Promotion of Science (http://www. jsps.go.jp/english/). The study was also supported by a Health and Labour Sciences Research Grant, and grants for Comprehensive Research on Aging and Health (H22-Choju-Shitei-008, H24-Junkankitou-Ippan-007, H24-Chikyukibo-Ippan-009, H24-ChojuWakate-009, H25-Kenki-Wakate-015, H25-Irryo-Shitei-003 [Fukkou], H26-Choju-Ippan-006, H27-Ninchisyou-Ippan-001, H28-Choju-Ippan-002) from the Ministry of Health, Labour and Welfare, Japan (http://www.mhlw.go.jp/english/), the Research and Development Grants for Longevity Science from AMED (Japan Agency for Medical Research and development, http://www. amed.go.jp/en/program/), the Personal Health Record (PHR) Utilization Project from AMED, World Health Organization Centre for Health Development (WHO Kobe Centre, http://www.who. int/kobe_centre/en/) (WHO APW 2017/713981), Japan Foundation for Aging and Health Research Support Grant (https://www. tyojyu.or.jp/en/), a grant from The Health Care Science Institute (http://www.iken.org/en/index.html), as well as grants from National Center for Geriatrics and Gerontology (http://www.ncgg. go.jp/english/). The research funding bodies had no role in the study design, data collection, data analysis, data interpretation, writing, or submitting of the report.

\section{Disclosure Statement}

The authors have no conflicts of interest to declare.

\section{References}

1 World Health Organization: World Health Statistics 2016. Geneva, World Health Organization, 2016

2 Ikeda N, Saito E, Kondo N, Inoue M, Ikeda S, Satoh T, Wada K, Stickley A, Katanoda K, Mizoue T, Noda M, Iso H, Fujino Y, Sobue T, Tsugane S, Naghavi M, Ezzati M, Shibuya K: What has made the population of Japan healthy? Lancet 2011;378:1094-1105.

3 Murray CJ: Why is Japanese life expectancy so high? Lancet 2011;378:1124-1125.

4 Marmot MG, Syme SL, Kagan A, Kato H, Cohen JB, Belsky J: Epidemiologic studies of coronary heart disease and stroke in Japanese men living in Japan, Hawaii and California: prevalence of coronary and hypertensive heart disease and associated risk factors. Am J Epidemiol 1975;102:514-525.

5 Marmot MG, Smith GD: Why are the Japanese living longer? BMJ 1989;299:1547-1551.

6 Manton KG, Vaupel JW: Survival after the age of 80 in the United States, Sweden, France, England, and Japan. N Engl J Med 1995;333: 1232-1235.
7 Stenholm S, Head J, Kivimaki M, Kawachi I, Aalto V, Zins M, Goldberg M, Zaninotto P, Magnuson Hanson L, Westerlund H, Vahtera J: Smoking, physical inactivity and obesity as predictors of healthy and disease-free life expectancy between ages 50 and 75: a multicohort study. Int J Epidemiol 2016;45:12601270.

8 Steptoe A, Breeze E, Banks J, Nazroo J: Cohort profile: the English longitudinal study of ageing. Int J Epidemiol 2013;42:1640-1648.

9 Marmot M, Oldfield Z, Clemens S, Blake M, Phelps A, Nazroo J, Steptoe A, Rogers N, Banks J, Oskala A: English Longitudinal Study of Ageing: Waves 0-7, 1998-2015. UK Data Service, 2017.

10 Nishi A, Kondo K, Hirai H, Kawachi I: Cohort profile: the AGES 2003 Cohort Study in Aichi, Japan. J Epidemiol 2011;21:151-157.

11 Tani Y, Kondo N, Nagamine Y, Shinozaki T, Kondo K, Kawachi I, Fujiwara T: Childhood socioeconomic disadvantage is associated with lower mortality in older Japanese men: the JAGES cohort study. Int J Epidemiol 2016; 45:1226-1235.
12 Poulain M, Herm A, Pes G: The Blue Zones: areas of exceptional longevity around the world. Vienna Yearb Popul Res 2013;11:87108.

13 The Global BMI Mortality Collaboration: Body-mass index and all-cause mortality: individual-participant-data meta-analysis of 239 prospective studies in four continents. Lancet 2016;388:776-786.

14 Rizzuto D, Orsini N, Qiu C, Wang HX, Fratiglioni L: Lifestyle, social factors, and survival after age 75: population based study. BMJ 2012;345:e5568.

15 Orsini N, Wolk A, Bottai M: Evaluating percentiles of survival. Epidemiology 2012;23: 770-771.

16 Bellavia A, Tektonidis TG, Orsini N, Wolk A, Larsson SC: Quantifying the benefits of Mediterranean diet in terms of survival. Eur J Epidemiol 2016;31:527-530.

17 Bellavia A, Larsson SC, Bottai M, Wolk A, Orsini N: Fruit and vegetable consumption and all-cause mortality: a dose-response analysis. Am J Clin Nutr 2013;98:454-459. 
18 Nur U, Shack LG, Rachet B, Carpenter JR, Coleman MP: Modelling relative survival in the presence of incomplete data: a tutorial. Int J Epidemiol 2010;39:118-128.

19 Rubin DB, Schenker N: Multiple imputation for interval estimation from simple random samples with ignorable nonresponse. J Am Stat Assoc 1986;81:366-374.

20 Vandenbroucke JP, von Elm E, Altman DG, Gotzsche PC, Mulrow CD, Pocock SJ, Poole C, Schlesselman JJ, Egger M, Initiative S: Strengthening the Reporting of Observational Studies in Epidemiology (STROBE): explanation and elaboration. PLoS Med 2007;4:e297.

21 Fagerlin A, Peters E: Quantitative Information; in Fischhoff B (ed): Communicating Risks and Benefits: An Evidence Based User's Guide. Silver Spring, Government Printing Office, 2012.

22 Ikram UZ, Malmusi D, Juel K, Rey G, Kunst AE: Association between integration policies and immigrants' mortality: an explorative study across three European countries. PLoS One 2015;10:e129916.

23 Karalexi MA, Papathoma P, Thomopoulos TP, Ryzhov A, Zborovskaya A, Dimitrova N, Zivkovic S, Eser S, Antunes L, Sekerija M, Zagar T, Bastos J, Demetriou A, Agius D, Cozma R, Coza D, Bouka E, Dessypris N, Belechri M, Dana H, Hatzipantelis E, Papakonstantinou E, Polychronopoulou S, Pourtsidis A, Stiakaki E, Chatziioannou A, Manolitsi K, Orphanidis G, Papadopoulos S, Papathanasiou M, Patsouris E, Sgouros S, Zountsas B, Moschovi M, Steliarova-Foucher E, Petridou ET: Childhood central nervous system tumour mortality and survival in Southern and Eastern Europe (1983-2014): gaps persist across 14 cancer registries. Eur J Cancer 2015;51:2665-2677.

24 Vaupel JW, Zhang Z, van Raalte AA: Life expectancy and disparity: an international comparison of life table data. BMJ Open 2011; 1:e000128.

25 Oksuzyan A, Crimmins E, Saito Y, O’Rand A, Vaupel JW, Christensen K: Cross-national comparison of sex differences in health and mortality in Denmark, Japan and the US. Eur J Epidemiol 2010;25:471-480.
26 Mossialos E, Djordjevic A, Osborn R, Sarnak D: International profiles of health care systems. The Commonwealth Fund, 2017.

27 Naimi TS, Stockwell T, Zhao J, Xuan Z, Dangardt F, Saitz R, Liang W, Chikritzhs T: Selection biases in observational studies affect associations between "moderate" alcohol consumption and mortality. Addiction 2017;112: 207-214.

28 Gmel G, Gutjahr E, Rehm J: How stable is the risk curve between alcohol and all-cause mortality and what factors influence the shape? A precision-weighted hierarchical meta-analysis. Eur J Epidemiol 2003;18:631-642.

29 Stockwell T, Zhao J, Panwar S, Roemer A, Naimi T, Chikritzhs T: Do "moderate" drinkers have reduced mortality risk? A systematic review and meta-analysis of alcohol consumption and all-cause mortality. J Stud Alcohol Drugs 2016;77:185-198.

30 Evans-Polce RJ, Staff J, Maggs JL: Alcohol abstention in early adulthood and premature mortality: do early life factors, social support, and health explain this association? Soc Sci Med 2016;163:71-79.

31 Zheng W, McLerran DF, Rolland B, Zhang X, Inoue M, Matsuo K, He J, Gupta PC, Ramadas K, Tsugane S, Irie F, Tamakoshi A, Gao YT, Wang R, Shu XO, Tsuji I, Kuriyama S, Tanaka H, Satoh H, Chen CJ, Yuan JM, Yoo KY, Ahsan H, Pan WH, Gu D, Pednekar MS, Sauvaget C, Sasazuki S, Sairenchi T, Yang G, Xiang YB, Nagai M, Suzuki T, Nishino Y, You SL, Koh WP, Park SK, Chen Y, Shen CY, Thornquist M, Feng Z, Kang D, Boffetta P, Potter JD: Association between body-mass index and risk of death in more than 1 million Asians. N Engl J Med 2011;364:719-729.

32 Nakade M, Takagi D, Suzuki K, Aida J, Ojima $\mathrm{T}$, Kondo K, Hirai H, Kondo N: Influence of socioeconomic status on the association between body mass index and cause-specific mortality among older Japanese adults: the AGES Cohort Study. Prev Med 2015;77:112118.

33 Peter RS, Mayer B, Concin H, Nagel G: The effect of age on the shape of the BMI-mortality relation and BMI associated with minimum all-cause mortality in a large Austrian cohort. Int J Obes (Lond) 2015;39:530-534.
34 Winter JE, MacInnis RJ, Wattanapenpaiboon $\mathrm{N}$, Nowson CA: BMI and all-cause mortality in older adults: a meta-analysis. Am J Clin Nutr 2014;99:875-890.

35 Fried LP, Tangen CM, Walston J, Newman AB, Hirsch C, Gottdiener J, Seeman T, Tracy R, Kop WJ, Burke G, McBurnie MA; Cardiovascular Health Study Collaborative Research G: Frailty in older adults: evidence for a phenotype. J Gerontol A Biol Sci Med Sci 2001; 56:M146-M156.

36 Chang SF, Lin PL: Frail phenotype and mortality prediction: a systematic review and meta-analysis of prospective cohort studies. Int J Nurs Stud 2015;52:1362-1374.

37 Murakami Y, Miura K, Okamura T, Ueshima H; EPOCH-JAPAN Research Group: Population attributable numbers and fractions of deaths due to smoking: a pooled analysis of 180,000 Japanese. Prev Med 2011;52:60-65.

38 Manzoli L, Villari P, M Pirone G, Boccia A: Marital status and mortality in the elderly: a systematic review and meta-analysis. Soc Sci Med 2007;64:77-94.

39 Holt-Lunstad J, Smith TB, Layton JB: Social relationships and mortality risk: a meta-analytic review. PLoS Med 2010;7:e1000316.

40 Marmot M: Social determinants of health inequalities. Lancet 2005;365:1099-1104.

41 Vathesatogkit P, Batty GD, Woodward M: Socioeconomic disadvantage and disease-specific mortality in Asia: systematic review with meta-analysis of population-based cohort studies. J Epidemiol Community Health 2014;68:375-383.

42 Hikichi H, Kondo N, Kondo K, Aida J, Takeda T, Kawachi I: Effect of a community intervention programme promoting social interactions on functional disability prevention for older adults: propensity score matching and instrumental variable analyses, JAGES Taketoyo study. J Epidemiol Community Health 2015;69:905-910.

43 Lund R, Christensen U, Nilsson CJ, Kriegbaum M, Hulvej Rod N: Stressful social relations and mortality: a prospective cohort study. J Epidemiol Community Health 2014; 68:720-727.
Differences in Survival among Older

Adults in Japan and England
Gerontology 2018;64:266-277

DOI: $10.1159 / 000485797$ 